\title{
Ein neuer adaptiver Prädiktor für die DPCM-Codierung von Fernseh- signalen
}

\author{
A New Adaptive Predictor for DPCM Coding of TV Signals
}

\author{
Von Chun-tian Zhang \\ Mitteilung aus dem Institut für Theoretische Nachrichtentechnik und Informationsverarbeitung der \\ UNIVERSITÄT HANNOVER
}

Übersicht:

Nichtadaptive zweidimensionale Prädiktoren für die DPCM-Codierung von Fernsehsignalen führen bei Kanten in Bildern meistens zu großen Prädiktionsfehlern und sind deshalb ungünstig für konturreiche Bilder. Ein neuer adaptiver Prädiktor wird vorgeschlagen, bei dem Bildbereiche mit geringen Signaländerungen und solche mit Konturen getrennt mit zugehörigen Prädiktoren verarbeitet werden. Die Güte des neuen Prädiktors wird mit bekannten nichtadaptiven und adaptiven Prädiktoren anhand von Computersimulationen verglichen. Die Vergleiche basieren sowohl auf quantitativen Kriterien als auch auf subjektiven Tests. Durch den vorgeschlagenen Prädiktionsalgorithmus wird speziell die Häufigkeit großer Prädiktionsfehler bei konturreichen Bildern erheblich reduziert. Verglichen mit anderen Prädiktionsalgorithmen führt dies zu einer geringeren Übertragungsbitrate bei gleicher Bildqualitāt.

\section{Abstract:}

Nonadaptive two-dimensional predictors for DPCM coding of TV signals exhibit large prediction errors at edges in pictures. Therefore they are unfavorable for high detailed pictures with many edges. A new adaptive predictor is proposed, whereby flat areas and edges of a picture are processed with different predictors. The gain of the new predictor is compared with known nonadaptive and adaptive predictors by means of computer simulations. The comparisons base on quantitative criteria as well as subjective tests. An advantage of the presented prediction algorithm is the considerable reduction of the frequency of large prediction errors in pictures with many edges. Compared to other prediction algorithms, this allows a reduction of the transmission rate.

Für die Dokumentation:

Adaptive DPCM / Adaptiver Prädiktor / Bildcodierung / Digitale Fernsehsignalcodierung

\section{Einführung}

Die Differenzpulscodemodulation (DPCM) ist ein einfach zu realisierendes Codierungsverfahren für die Reduktion der Übertragungsbitrate von digitalen Bildsignalen. Bild 1 zeigt das Blockschaltbild eines DPCM-Systems. Hierbei wird von jedem Abtastwert $s$ ein Prädiktionswert $\hat{s}$ abgezogen. Die Differenz (Prädiktionsfehler)

$$
e=s-\hat{s}
$$

wird quantisiert, codiert und übertragen. Beim Empfänger wird nach der Decodierung der quantisierte Prädiktionsfehler $e^{\prime}$ zu dem Prädiktionswert $\hat{s}$ addiert, und man erhält dann den rekonstruierten Abtastwert

$$
\boldsymbol{s}^{\prime}=\boldsymbol{e}^{\prime}+\hat{\boldsymbol{s}} \text {. }
$$

Da nur vorher übertragene rekonstruierte Abtastwerte beim Sender und beim Empfänger zur Ermittlung des Prädiktionswertes benutzt werden, wird bei beiden Seiten der gleiche Prädiktionswert $\hat{s}$ bestimmt, sofern der Übertragungskanal ungestört ist. Es ist leicht zu zeigen, daß der Quantisierungsfehler des Prädiktionsfehlers gleich dem Fehler des rekonstruierten Signals ist:

$$
q=e^{\prime}-e=s^{\prime}-s .
$$

Ein Prädiktor ist so zu wählen, daß möglichst nur kleine Prädiktionsfehler auftreten. Die Entropie des zu übertragenden Prädiktionsfehlers $e$ ist in diesem Fall wesentlich kleiner als die Entropie des Eingangssignales $s$. Dies führt auf eine geringere Übertragungsbitrate. Als Kriterium für die Optimierung von Prädiktoren wird vielfach die minimale Varianz des Prädiktionsfehlers wegen der Beziehung zur Leistung und der güristigent

mathematischen Handhabung benutzt [1-3]. Die rekursive Struktur der DPCM führt auf eine gegenseitige Beeinflussung von Prädiktor und Quantisierer. Unter Berücksichtigung dieses Effektes hat Pirsch [4] einen Prädiktor mit nur positiven Koeffizienten vorgeschlagen, welcher den Einfluß der Quantisierungsfehlerrückkopplung reduziert. Ein solcher Prädiktor führt auf vergleichsweise weniger wahrnehmbare Quantisierungsfehler.

Dem nichtadaptiven Prädiktor liegt das Modell einer stationären Signalquelle zugrunde, jedoch sind die tatsächlichen Fernsehsignale im allgemeinen als räumlich nichtstationär zu betrachten. Ein nichtadaptiver Prädiktor
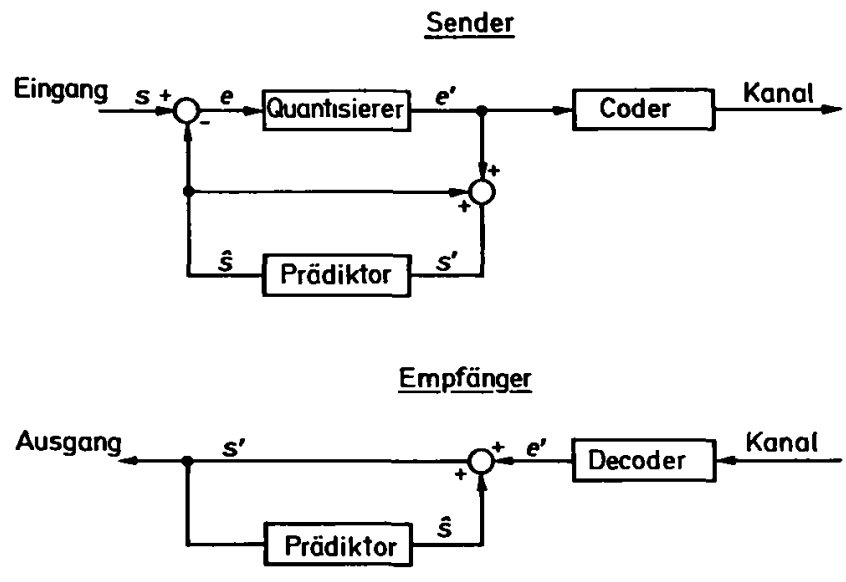

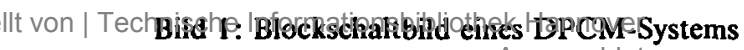


führt meistens zu großen Prädiktionsfehlern bei Konturen und Kanten in Bildern. Die an Konturen und Kanten erzeugten großen Prädiktionsfehler verursachen spezielle Quantisierungseffekte, die als ,edge busyness“ und „slope overload" bezeichnet werden. Dies kann insbesondere bei Testbildern mit konturreicher Auflösung beobachtet werden. Slope overload führt bei diesen Bildern nicht nur zu Unschärfe der rekonstruierten Kanten, sondern auch zu Moiré-Mustern. Um Moiré-Störungen zu verhindern, benötigt man für Auflösungstestbilder mehr Quantisierungsstufen als für natürliche Bilder [4].

Eine Verbesserung dieses Problems kann durch umschaltbare Prädiktoren erreicht werden, welche sich adaptiv den lokalen Eigenschaften von Bildern anpassen. Auf der Basis der Konturprädiktion wurde von Zschunke [5, 6] ein adaptiver Prädiktor vorgeschlagen. Im Vergleich $\mathbf{z u}$ den festen Prädiktoren führt ein solcher Prädiktor nur bei Auflösungstestbildern zu einer verbesserten Vorhersage. Bei natürlichen Bildern wird aber eine Verschlechterung festgestellt. Musmann [7] hat darauf hingewiesen, daB bei zukünftigen Untersuchungen die durch Prädiktorumschaltungen verursachten Quantisierungseffekte zu berücksichtigen sind.

Unter Berücksichtigung der Ergebnisse von [4, 5, 7] wird hier ein neuer adaptiver Prädiktor für die DPCMIntraframe-Codierung von Schwarz-Weiß-Fernsehsignalen mit einer Bandbreite von $5 \mathrm{MHz}$ vorgeschlagen. Gebiete mit geringen Signaländerungen und Konturen eines Bildes werden voneinander getrennt mit zugehörigen Prädiktoren verarbeitet. Die Vorzüge der Prädiktoren von Pirsch [4] und Zschunke [5] werden hierbei berücksichtigt. Die Zielsetzung für den neuen adaptiven Prädiktor kann wie folgt beschrieben werden:

- Verbesserung der Prädiktion für Auflösungstestbilder, ohne die Prädiktion bei natürlichen Bildern zu verschlechtern;

- Verringerung der Häufigkeit großer Prädiktionsfehler, um edge busyness und slope overload möglichst $\mathrm{zu}$ vermeiden.

Im folgenden Kapitel wird der Algorithmus des neuen Prädiktors beschrieben. Basierend auf Computersimulationen werden Vergleiche mit bekannten Prädiktoren durchgeführt.

\section{Prädiktionsalgorithmus}

\subsection{Symbole und Definitionen}

Nachfolgend werden die Signale sowohl durch Bildkoordinaten (z. B.: $s(i, j)$ ) als auch durch die relative Lage zum aktuellen Bildpunkt beschrieben (z. B.: $s_{\text {s }}$ ). Bild 2 zeigt die hier benutzte relative Indizierung. Es gelten folgende Symbole und Definitionen:

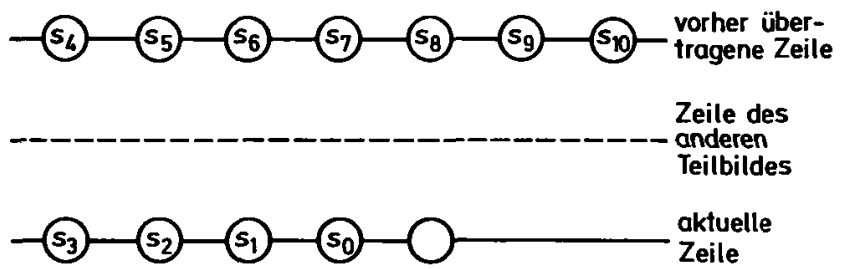

Bild 2: Abtastraster und Bezeichnung der Abtastwerte $s_{0} \quad$ Luminanzwert des aktuellen Bildpunkts

$s_{i} \quad i=1, \ldots, N$; Luminanzwerte vorangegangener Bildpunkte

$s_{i}^{\prime} \quad i=1, \ldots, N$; rekonstruierte Luminanzwerte

$\hat{\boldsymbol{s}} \quad$ Prädiktionswert von $s_{0}$

$D_{i j} \quad$ Differenz zwischen $s_{i}^{\prime}$ und $s_{j}^{\prime}, D_{i j}=s_{i}^{\prime}-s_{j}^{\prime}$

$\left|D_{i j}\right|$ Betrag von $D_{i j}$

a Schwellwert ( $a=0 \ldots 255)$

$V D_{i j}$ Vorzeichenfunktion von $D_{i j} \mathrm{mit}$

$$
\begin{aligned}
& V D_{i j}=\left\{\begin{array}{rrrr}
1 & \text { für } & D_{i j} \geq & a \\
0 & \text { für } & -a<D_{i j}< & a \\
-1 & \text { für } & D_{i j} \leq-a
\end{array}\right. \\
& V_{12 i j}=\left\{\begin{array}{lll}
V D_{12} V D_{i j} & \text { für } & V D_{12} \neq 0 \\
V D_{23} V D_{i j} & \text { für } & V D_{12}=0
\end{array}\right.
\end{aligned}
$$

\subsection{Konturdetektion}

a) Ein Konturprädiktor basierend auf Konturextrapolation ist nur für lange und steile Konturen günstig [5]. Ein solcher Prädiktor bringt für natürliche Bilder keinen Gewinn. Aus diesem Grund ist es sinnvoll, Gebiete mit geringen Signaländerungen und Konturen eines Bildes getrennt mit verschiedenen Prädiktoren zu verarbeiten. Hierzu wird das Kriterium

$$
F L K R=\operatorname{Max}\left(\left|D_{12}\right|,\left|D_{15}\right|,\left|D_{16}\right|,\left|D_{17}\right|\right)
$$

vorgeschlagen. Ist $F L K R<20$, wird die Umgebung des aktuellen Bildpunktes als flach betrachtet und ein zweidimensionaler Prädiktor mit nur positiven Koeffizienten verwendet.

b) Die Konturrichtung des Bildpunktes $s_{0}$ kann aus der Konturrichtung des vorhergehenden benachbarten Bildpunktes $s_{1}$ entnommen werden. Bei der Feststellung der Konturrichtung wird zuerst die horizontale Richtung überprüft. Dies erfolgt durch einen Vergleich der beiden nachfolgenden Parameter:

$$
\begin{aligned}
& D V=\operatorname{Min}\left(\left|D_{15}\right|,\left|D_{16}\right|,\left|D_{17}\right|,\left|D_{18}\right|\right) \\
& D H=\operatorname{Max}\left(\left|D_{12}\right|,\left|D_{23}\right|\right) .
\end{aligned}
$$

Ist $D H<D V$, so nehmen wir an, daß eine horizontale Kontur vorliegt, weil die Amplitudenānderung des Abtastwertes in dieser Richtung minimal ist. Es wird nicht nur $\left|D_{12}\right|$, sondern auch $\left|D_{23}\right|$ überprüft, weil das Abtastintervall in der horizontalen Richtung kleiner als in vertikaler Richtung ist.

c) Die in nichthorizontaler Richtung durch den Bildpunkt $s_{1}$ gehende Kontur kann durch die Position eines Nachbarbildpunktes von $s_{1}$ festgestellt werden, welcher die kleinste Amplitudendifferenz $\mathrm{zu} s_{1}$ hat und mit $s_{1}$ auf der gleichen Kantenseite liegt. Auf welcher Kantenseite ein Bildpunkt liegt, wird durch die Vorzeichenfunktion $V D_{i j}$ der Amplitudendifferenz zwischen diesem Punkt und einem in der gleichen Zeile benachbarten Bezugsbildpunkt ermittelt. Gemäß der Position des betrachteten Bildpunktes wird der linke oder der rechte von beiden Nachbarbildpunkten als solcher Bezugspunkt ausgewählt (s. Abschnitt $2.3 \mathrm{~d}$ ). Ungewöhnlich ist, daß das Vorzeichen $V D_{i j}$ als 0 definiert wird, wenn der Betrag von $\left|D_{i j}\right|$ unter einer Schwelle von z. B. $a=7$ liegt. In diesem Fall ist der Zustand undefiniert, und der betrachtete Bildpunkt darf nicht zur Bestimmung der Konturrichtung benutzt werden, womit eine durch Quantisierungsfehler oder Signalquellenrauschen verursachte falsche Konturprädiktion verhindert

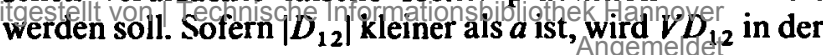


Definition von $V_{12 i j}$ durch $V D_{23}$ ersetzt. Dadurch erhält man auch für $V D_{12}=0$ noch eine Chance für die Konturprädiktion. Wie Untersuchungen bestätigen, kann ein adaptiver Konturprädiktor zu vielen kleinen Prädiktionsfehlern führen, allerdings auch viele große Fehler verursachen, sofern sich die aktuelle Konturrichtung zu stark ändert und nicht vorhergesagt werden kann. Deswegen sollen die Prädiktorumschaltungen zwischen Nachbarkonturrichtungen abgeschwächt werden. Statt eines einzelnen Bildpunkts wird deshalb in unserem Algorithmus jeweils eine Kombination von zwei oder noch mehr Bildpunkten zur Ermittlung des Prädiktionswertes benutzt.

d) Schwankt die Signalamplitude in der Umgebung des aktuellen Bildpunkts sehr stark, so nehmen wir an, daß solche Bildteile zu Texturen gehören. Es wird beobachtet, daß wegen Störungen durch Quantisierungsfehler und Kamerarauschen ein Konturprädiktor bei Texturen oft zu Unruhe in der rekonstruierten Bildsequenz führt. Aus diesem Grund unterbrechen wir bei solchen Bildgebieten die Konturprädiktion und verwenden als Prädiktionswert den Mittelwert von fünf Nachbarbildpunkten (s. Abschnitt 2.3c).

e) Befindet sich überhaupt kein Bildpunkt mit dem Bildpunkt $s_{1}$ auf der glëichen Kantenseite oder überschreitet die bei der Konturfindung erhaltene minimale Amplitudendifferenz zwischen $s_{1}$ und dessen Nachbarbildpunkten eine Schwelle, z. B. 50, so nehmen wir an, daß eine starke Änderung oder Beugung der Konturrichtung auftritt. In diesem Fall wird der in a) erwähnte zweidimensionale Prädiktor mit nur positiven Koeffizienten benutzt.

\subsection{Algorithmus}

Der Algorithmus ist durch die nachfolgend beschriebenen Schritte definiert, wobei der jeweils folgende Schritt nur ausgeführt wird, wenn alle vorhergehenden Bedingungen nicht erfüllt sind.

a) Ist $F L K R<20$, gilt

$$
\hat{s}=\frac{5}{8} s_{1}^{\prime}+\frac{1}{8}\left(s_{6}^{\prime}+s_{7}^{\prime}+s_{8}^{\prime}\right) .
$$

b) Ist $D H<D V$, gilt

$$
\hat{s}=\frac{3}{4} s_{1}^{\prime}+\frac{1}{4} s_{7}^{\prime} .
$$

c) Ist eine der vier folgenden Bedingungen erfüllt, gilt

$$
\hat{s}=\frac{1}{5}\left(s_{5}^{\prime}+s_{6}^{\prime}+s_{7}^{\prime}+s_{8}^{\prime}+s_{9}^{\prime}\right) \text {. }
$$

Die Bedingungen sind:

$\begin{array}{lllll}\text { c1) } V_{1254} \neq-1 & \text { und } V_{1265}=-1 & \text { und } & V_{1276} \neq-1 ; \\ \text { c2) } V_{1254} \neq-1 & \text { und } V_{1265}=-1 & \text { und } & V_{1287} \neq-1 ; \\ \text { c3) } V_{1254} \neq-1 & \text { und } V_{1276}=-1 & \text { und } & V_{1287} \neq-1 ; \\ \text { c4) } V_{1265} \neq-1 & \text { und } & V_{1276}=-1 & \text { und } & V_{1287} \neq-1\end{array}$

d) Es sei

$$
\begin{aligned}
& A_{5}= \begin{cases}\left|D_{15}\right| & \text { wenn } V_{1265}=1, \\
255 & \text { sonst; }\end{cases} \\
& A_{6}= \begin{cases}\left|D_{16}\right| & \text { wenn } V_{1265} \text { oder } V_{1276}=1, \\
255 & \text { sonst; }\end{cases}
\end{aligned}
$$

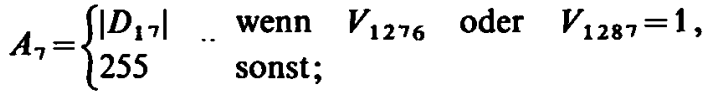

$A_{8}= \begin{cases}\left|D_{18}\right| & \text { wenn } \quad V_{1287}=1, \\ 255 & \text { sonst; }\end{cases}$

und

$$
D K=\operatorname{Min}\left(A_{5}, A_{6}, A_{7}, A_{8}\right) .
$$

d1) Ist $D K>50$, gilt

$$
\hat{s}=\frac{5}{8} s_{1}^{\prime}+\frac{1}{8}\left(s_{6}^{\prime}+s_{7}^{\prime}+s_{8}^{\prime}\right) .
$$

d2) Ist $D K=A_{i}$, gilt

$$
\hat{s}=\frac{1}{4} s_{i}^{\prime}+\frac{1}{2} s_{i+1}^{\prime}+\frac{1}{4} s_{i+2}^{\prime} \quad \text { mit } \quad i=5,6,7,8 .
$$

\section{Vergleiche mit anderen Prädiktoren}

\subsection{Prädiktoren}

Die folgenden drei Prädiktoren wurden zu Vergleichen herangezogen.

Prädiktor 1: Vorschlag von Pirsch [4]

$$
\hat{s}=\frac{1}{2} s_{1}^{\prime}+\frac{1}{8} s_{6}^{\prime}+\frac{1}{4} s_{7}^{\prime}+\frac{1}{8} s_{8}^{\prime} .
$$

Prädiktor 2: ein nach dem Kriterium minimale Varianz bestimmter Prädiktor

$$
\hat{s}=\frac{7}{8} s_{1}^{\prime}-\frac{5}{8} s_{6}^{\prime}+\frac{6}{8} s_{7}^{\prime} .
$$

Prädiktor 3: ein von Zschunke [5] vorgeschlagener Konturprädiktor. Der Algorithmus wird nachfolgend mit den Symbolen des Abschnitts 2.1 beschrieben.

a) Ist $D_{12}<26$, gilt

$$
\hat{s}=s_{1}^{\prime} .
$$

b) Es sei

$A_{5}=\left\{\begin{array}{ll}\left|D_{15}\right| & \text { wenn } \\ 255 & \text { sonst; }\end{array} \quad V D_{12}=V D_{54}\right.$,

$A_{6}=\left\{\begin{array}{ll}\left|D_{16}\right| & \text { wenn } \\ 255 & \text { sonst; }\end{array} \quad V D_{12}=V D_{65}\right.$,

$A_{7}= \begin{cases}\left|D_{17}\right| & \text { wenn } V D_{12}=V D_{76}, \\ 255 & \text { sonst; }\end{cases}$

Schwelle $a=4$ für $V D_{i j}$

und

$$
D K=\operatorname{Min}\left(A_{5}, A_{6}, A_{7}\right) .
$$

b1) Ist $D K>64$, gilt

$$
\text { b2) Ist } D K=A_{i} \text {, gilt } \begin{aligned}
& \hat{s}=s_{1}^{\prime} . \\
& \hat{s}=s_{i+1}^{\prime}
\end{aligned} \text { mit } i=5,6,7 .
$$

Der neu vorgeschlagene adaptive Prädiktor wird als Prädiktor 4 bezeichnet.

\subsection{Objektive Vergleichskriterien}

Insgesamt wurden vier objektive Kriterien für den Vergfeich der Pradiktoren benutzt. 
A

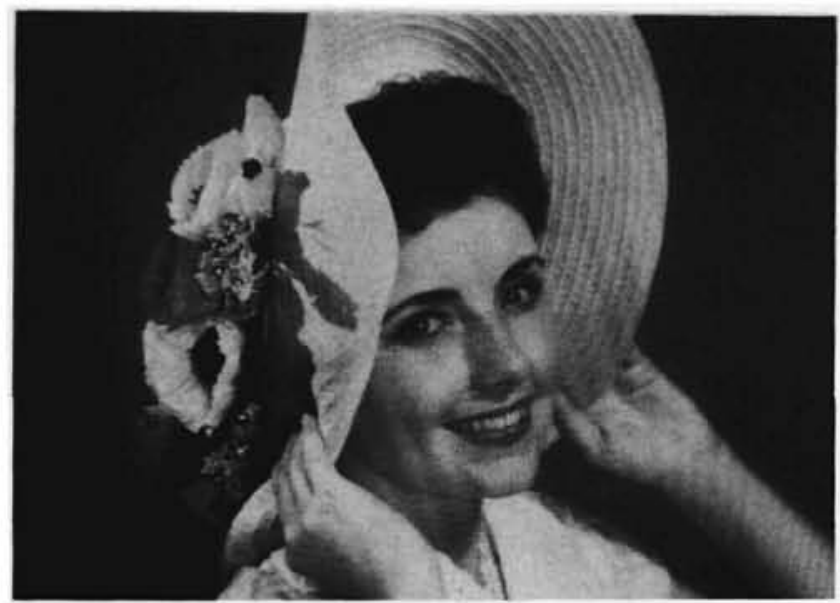

B

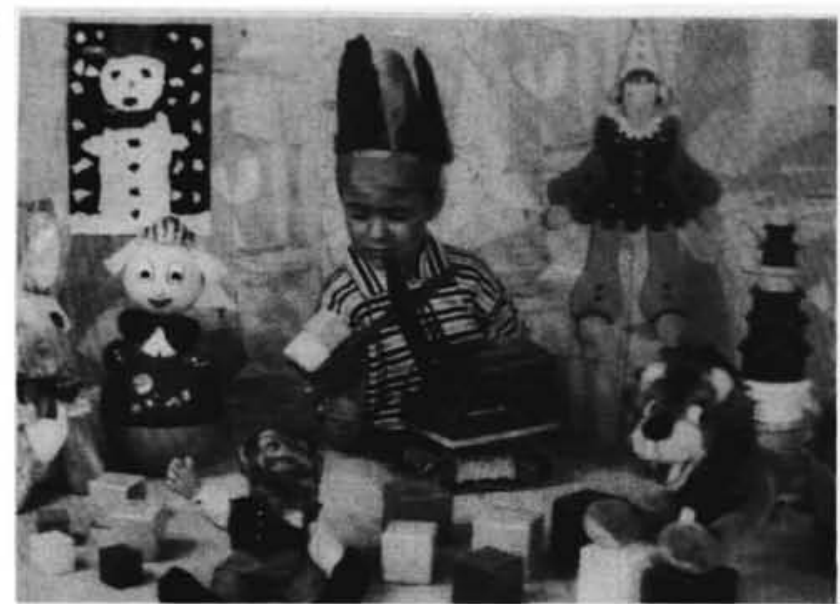

$\mathrm{C}$

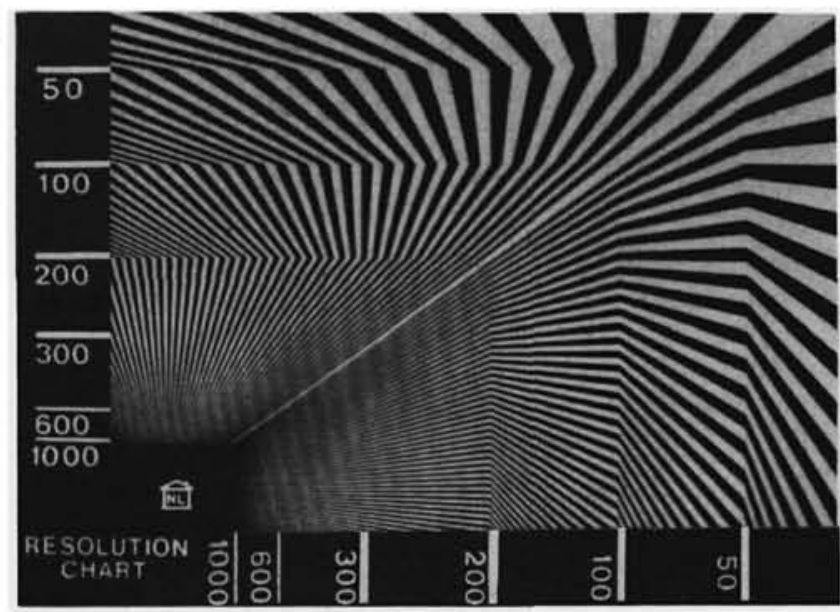

Bild 3: Testbilder

A Strohhutdame

B Spielzeugjunge

C Auflösungstestbild

$$
\begin{aligned}
& -65,-50,-39,-30,-21,-14,-7,-2 \text {, } \\
& \text { 3. } 8,15,22,31,40,51,66
\end{aligned}
$$

Tabelle 1: Repräsentativwerte der 16-Stufen-Quantisierungscharakteristik a) Spitzenwert $e_{\mathrm{p}}$ des Prädiktionsfehlers: Der Prädiktionsfehler $e(i, j)$ ist definiert als

$$
e(i, j)=s(i, j)-\hat{s}(i, j)
$$

mit $s(i, j)$ als dem $j$-ten Abtastwert von der $i$-ten Zeile und $\hat{s}(i, j)$ als der Prädiktionswert von $s(i, j)$.

Da das Eingangssignal entsprechend 8-bit-PCM quantisiert wurde, liegen alle Prädiktionsfehler im Bereich $(-255,255)$. Der Spitzenwert des Prädiktionsfehlers ist so definiert, daß $99 \%$ der Prädiktionsfehler innerhalb des Bereichs $\left(-e_{\mathrm{p}}, e_{\mathrm{p}}\right)$ liegen.

b) Standardabweichung $\sigma_{\mathrm{f}}$ der Prädiktionsfehler:

$$
\sigma_{\mathrm{f}}=\sqrt{\frac{1}{N} \sum_{i} \sum_{j}[s(i, j)-\hat{s}(i, j)]^{2}},
$$

wobei $N$ die Anzahl der Abtastwerte des verarbeiteten Bildes ist.

c) Entropie $H_{\mathrm{f}}$ der Prädiktionsfehler:

$$
H_{\mathrm{f}}=-\sum_{e=-255}^{+255} \frac{M(e)}{N} \log _{2}\left[\frac{M(e)}{N}\right]
$$

mit $M(e) / N$ als relative Häufigkeit des Prädiktionsfehlers e.

d) Standardabweichung $\sigma_{q}$ der Quantisierungsfehler:

$$
\sigma_{\mathrm{q}}=\sqrt{\frac{1}{N} \sum_{i} \sum_{j}\left[s(i, j)-s^{\prime}(i, j)\right]^{2}},
$$

wobei $s^{\prime}(i, j)$ der rekonstruierte Wert von $s(i, j)$ beim Empfänger ist.

\section{Simulationsergebnisse}

Computersimulationen mit drei Testbildern wurden auf einem CYBER 76 Computer des Regionalen Rechenzentrums Niedersachsen und am Bildverarbeitungssystem des Instituts für Theoretische Nachrichtentechnik und Informationsverarbeitung der Universität Hannover durchgeführt. Der zentrale ProzeBrechner dieses Bildverarbeitungssystems ist eine PDP 11/45. Die folgenden drei Testbilder standen für die Computersimulationen zur Verfügung (Bild 3):

Testbild A: Strohhutdame,

Testbild B: Spielzeugjunge,

Testbild C: Auflösungstestbild.

Als Eingangssignal wurde die Luminanz-Komponente des 5-MHz-Fernsehsignals mit $10 \mathrm{MHz}$ abgetastet und mit 8 bit pulscodemoduliert. Für die Untersuchungen wurden die Daten eines Bildausschnittes von 540 Zeilen und $\mathbf{4 8 0}$ Bildpunkten pro Zeile für jedes Testbild benutzt. Die subjektiven Tests wurden mit einer Sequenz von 16 aufeinanderfolgenden Teilbildern durchgeführt, um die typischen Rauscheffekte der DPCM sichtbar zu machen. Wegen der Beschränkung der Speicherkapazität des Bildverarbeitungssystems wurde in diesem Fall ein Bildausschnitt von $128 \times 128$ Bildpunkten pro Teilbild verwendet. Der Bildausschnitt wurde so gewählt, daß das kritischste Gebiet im Ausschnitt liegt.

Bei den Computersimulationen wurde bei allen Prädiktoren ein 4-bit-Quantisierer mit 16 Stufen (Tabelle 1) verwendet. Dieser Quantisierer hat eine Quantisierungsfehleritcharalkteristikç dieàneetwar demn woin/ Binsch [ [4] worgeschla- 


\begin{tabular}{|c|l|c|}
\hline & Blld & $H_{0}$ \\
\hline A & Strohhutdame & 7,33685 \\
\hline B & Splelzeugjunge & 7,49038 \\
\hline C & $\begin{array}{l}\text { Auflösungstest- } \\
\text { bild }\end{array}$ & 7,25794 \\
\hline
\end{tabular}

Tabelle 2: Entropie $H_{0}$ der PCM-Abtastwerte in bit/Abtastwert genen 13-Stufen-Quantisierer für natürliche Bilder entspricht. Es ist klar, daß der Quantisierer an sich für jeden Prädiktor separat zu bestimmen ist. Aber wenn man daran denkt, daß ein fester Quantisierer nicht für alle Bildarten optimal sein kann, soll zur Vereinfachung des Vergleiches für alle Prädiktoren der gleiche Quantisierer verwendet werden. Dies wird auch durch Simulationen bestätigt, die zeigen, daß der Quantisierer keinen bedeutenden Einfluß auf die Rangfolge der Prädiktoren hat.

\begin{tabular}{|c|c|c|c|c|c|c|}
\hline & \multicolumn{2}{|c}{ Strohhutaame } & \multicolumn{2}{c|}{ Spielzeugjunge } & \multicolumn{2}{c|}{ Auflösungstestbild } \\
\hline Prädiktor & $\begin{array}{c}16 \text {-Stufen- } \\
\text { Quantisierex }\end{array}$ & $\begin{array}{c}\text { ohne } \\
\text { Quantisierer }\end{array}$ & $\begin{array}{c}\text { 16-Stufen- } \\
\text { Quantisierer }\end{array}$ & $\begin{array}{c}\text { ohne } \\
\text { Quantisierer }\end{array}$ & $\begin{array}{c}\text { 16-Stufen- } \\
\text { Quantisierer }\end{array}$ & $\begin{array}{c}\text { ohne } \\
\text { Quantisierer }\end{array}$ \\
\hline 1 & 2,28831 & 4,61630 & 2,62882 & 5,03772 & 3,70054 & 6,62172 \\
\hline 2 & 2,42690 & 4,76078 & 2,57830 & 4,94027 & 3,74188 & 6,66530 \\
\hline 3 & 2,49578 & 4,86058 & 2,62986 & 5,03293 & 3,32976 & 6,04427 \\
\hline 4 & 2,24292 & 4,55677 & 2,49395 & 4,86735 & 3,52690 & 6,21502 \\
\hline
\end{tabular}

Tabelle 3: Entropic $H_{\mathrm{f}}$ der Prädiktionsfehler in bit/Abtastwert

\begin{tabular}{|c|c|c|c|c|c|c|c|c|c|c|c|c|}
\hline \multirow[b]{2}{*}{ Bild } & \multirow[b]{2}{*}{ Prädiktor } & \multicolumn{11}{|c|}{ Betragsbereich der Prädiktionsfehler } \\
\hline & & $0-9$ & $10-19$ & $20-29$ & $30-39$ & $40-49$ & $50-59$ & $60-69$ & $70-79$ & $80-89$ & $90-99$ & $100-$ \\
\hline \multirow{4}{*}{$\begin{array}{l}\text { Auflö- } \\
\text { sungs- } \\
\text { test- } \\
\text { bild }\end{array}$} & 1 & 0,473 & 0,114 & 0,092 & 0,080 & 0,071 & 0,065 & 0,052 & 0,036 & 0,014 & $2,98 \times 10^{-3}$ & $5.07 \times 10^{-4}$ \\
\hline & 2 & 0,478 & 0,145 & 0,107 & 0.079 & 0,065 & 0,048 & 0,032 & 0,021 & 0.013 & $7,03 \times 10^{-3}$ & $7,19 \times 10^{-3}$ \\
\hline & 3 & 0,643 & 0,121 & 0,060 & 0,043 & 0,036 & 0,032 & 0,026 & 0,018 & 0,012 & $6,11 \times 10^{-3}$ & $2,96 \times 10^{-3}$ \\
\hline & 4 & 0,540 & 0,152 & 0,119 & 0,089 & 0,052 & 0,027 & 0,012 & $5,71 \times 10^{-3}$ & $2,12 \times 10^{-3}$ & $7,89 \times 10^{-4}$ & $6,81 \times 10^{-4}$ \\
\hline \multirow{4}{*}{$\begin{array}{l}\text { Stroh- } \\
\text { hut- } \\
\text { dame }\end{array}$} & 1 & 0,880 & 0,090 & 0,020 & $6,42 \times 10^{-3}$ & $2,54 \times 10^{-3}$ & $9,47 \times 10^{-4}$ & $4,49 \times 10^{-4}$ & $1,83 \times 10^{-4}$ & $4,98 \times 10^{-5}$ & 0 & $8,31 \times 10^{-6}$ \\
\hline & 2 & 0,856 & 0,119 & 0,018 & $4,71 \times 10^{-3}$ & $1,42 \times 10^{-3}$ & $6,15 \times 10^{-4}$ & $2,58 \times 10^{-4}$ & $9,13 \times 10^{-5}$ & 0 & $8,31 \times 10^{-6}$ & $2,49 \times 10^{-5}$ \\
\hline & 3 & 0,845 & 0,113 & 0,028 & $8,03 \times 10^{-3}$ & $3,47 \times 10^{-3}$ & $1,79 \times 10^{-3}$ & $7,14 \times 10^{-4}$ & $3,16 \times 10^{-4}$ & $1,66 \times 10^{-4}$ & $6,65 \times 10^{-5}$ & $3,32 \times 10^{-5}$ \\
\hline & 4 & 0,887 & 0,087 & 0,016 & $4,63 \times 10^{-3}$ & $1,99 \times 10^{-3}$ & $7,48 \times 10^{-4}$ & $3,16 \times 10^{-4}$ & $1,74 \times 10^{-4}$ & $6,65 \times 10^{-5}$ & $3,32 \times 10^{-5}$ & 0 \\
\hline
\end{tabular}

Tabelle 4: Relative Häufigkeit des Prädiktionsfehlersbetrages bei 16-Stufen-Quantisierung nach Tabelle 1

\begin{tabular}{|c|c|c|c|}
\hline Pradiktor & Strohhutdame & $\begin{array}{c}\text { B1ld } \\
\text { Splel zeugjunge }\end{array}$ Auf losungstestbild \\
\hline 1 & 30 & 46 & 84 \\
\hline 2 & 32 & 39 & 95 \\
\hline 3 & 33 & 46 & 88 \\
\hline 4 & 27 & 39 & 68 \\
\hline
\end{tabular}

\subsection{Vergleiche mit objektiven Kriterien}

Die Entropie $\boldsymbol{H}_{0}$ der drei Bilder ist in Tabelle 2 angegeben. Tabelle 3 zeigt die Entropie $\boldsymbol{H}_{\mathrm{f}}$ der Prädiktionsfehler. Die Prädiktoren werden mit und ohne Quantisierer miteinander verglichen. Prädiktor 3 liefert für das Auflösungstestbild die größte Reduktion der Entropie. Das ist verständlich, wenn man die Prädiktionsfehlerverteilungen (Tabelle 4) betrachtet. Prädiktor 3 macht mehr kleine Prädiktionsfehler als die anderen. Aber für natürliche Bilder ist er eindeutig schlechter als feste Prädiktoren. Prädiktor 4 ist am günstigsten bei natürlichen Bildern und steht an der Tabelle 5: Spitzenwert $e_{p}$ des Prädiktionsfehlers bei 16-Stufen- zweiten Stelle beim Auflösungstestbild. Tabelle 4 zeigt die
Quantisierung nach Tabelle 1 Quantisierung nach Tabelle 1 


\begin{tabular}{|c|c|c|c|c|c|c|}
\hline & \multicolumn{2}{|c}{ Strohhutdame } & \multicolumn{2}{c|}{ Spielzeugjunge } & \multicolumn{2}{c|}{ Auflösungstestbild } \\
\hline Prädiktor & $\begin{array}{c}16 \text {-Stufen- } \\
\text { Quantisierer }\end{array}$ & $\begin{array}{c}\text { ohne } \\
\text { Quantisierer }\end{array}$ & $\begin{array}{c}16 \text {-Stufen- } \\
\text { Quantisierer }\end{array}$ & $\begin{array}{c}\text { ohne } \\
\text { Quantisierer }\end{array}$ & $\begin{array}{c}16 \text {-Stufen- } \\
\text { Quantisierer }\end{array}$ & $\begin{array}{c}\text { ohne } \\
\text { Quantisierer }\end{array}$ \\
\hline 1 & 7,64 & 7,57 & 11,16 & 11,03 & 32,56 & 31,85 \\
\hline 2 & 7,65 & 7,39 & 9,52 & 9,27 & 30,87 & 30,35 \\
\hline 3 & 8,87 & 8,73 & 10,88 & 10,76 & 25,81 & 25,76 \\
\hline 4 & 7,18 & 7,07 & 9,54 & 9,34 & 22,55 & 22,17 \\
\hline
\end{tabular}

Tabelle 6: Standardabweichung $\sigma_{\mathrm{f}}$ der Prädiktionsfehler

\begin{tabular}{|c|c|c|c|}
\hline Prädiktor & \multicolumn{3}{|c|}{$\begin{array}{c}\text { Bild } \\
\text { Strohhutdame }\end{array}$ Spielzeugjunge } \\
Aufiosungstestb1ld \\
\hline 1 & 1,56 & 1,77 & 3,96 \\
\hline 2 & 1,58 & 1,66 & 5,73 \\
\hline 3 & 1,65 & 1,86 & 4,46 \\
\hline 4 & 1,55 & 1,73 & 2,75 \\
\hline
\end{tabular}

lösungstestbild und beim konturarmen Bild Strohhutdame. Der Vorteil von Prädiktor 4 ist deutlich zu erkennen. Im Vergleich mit den anderen Prädiktoren führt Prädiktor 4 auf wesentlich weniger große Prädiktionsfehler. Beim Auflösungstestbild liefert Prädiktor 4 etwa $1 / 3$ weniger Prädiktionsfehler mit einem Betrag über $\mathbf{4 0}$ als Prädiktor 3. Gegenüber nichtadaptiven Prädiktoren beträgt der Unterschied etwa $2 / 3$. Dieser Vorzug von Prädiktor 4 bleibt auch bei konturarmen natürlichen Bildern für Prädiktionsfehler größer als 20 bestehen. Tabelle 5 zeigt den

Tabelle 7: Standardabweichung $\sigma_{\mathrm{q}}$ der Quantisierungsfehler Spitzenwert des Prädiktionsfehlers. Bei Prädiktor 4 ist die-

A
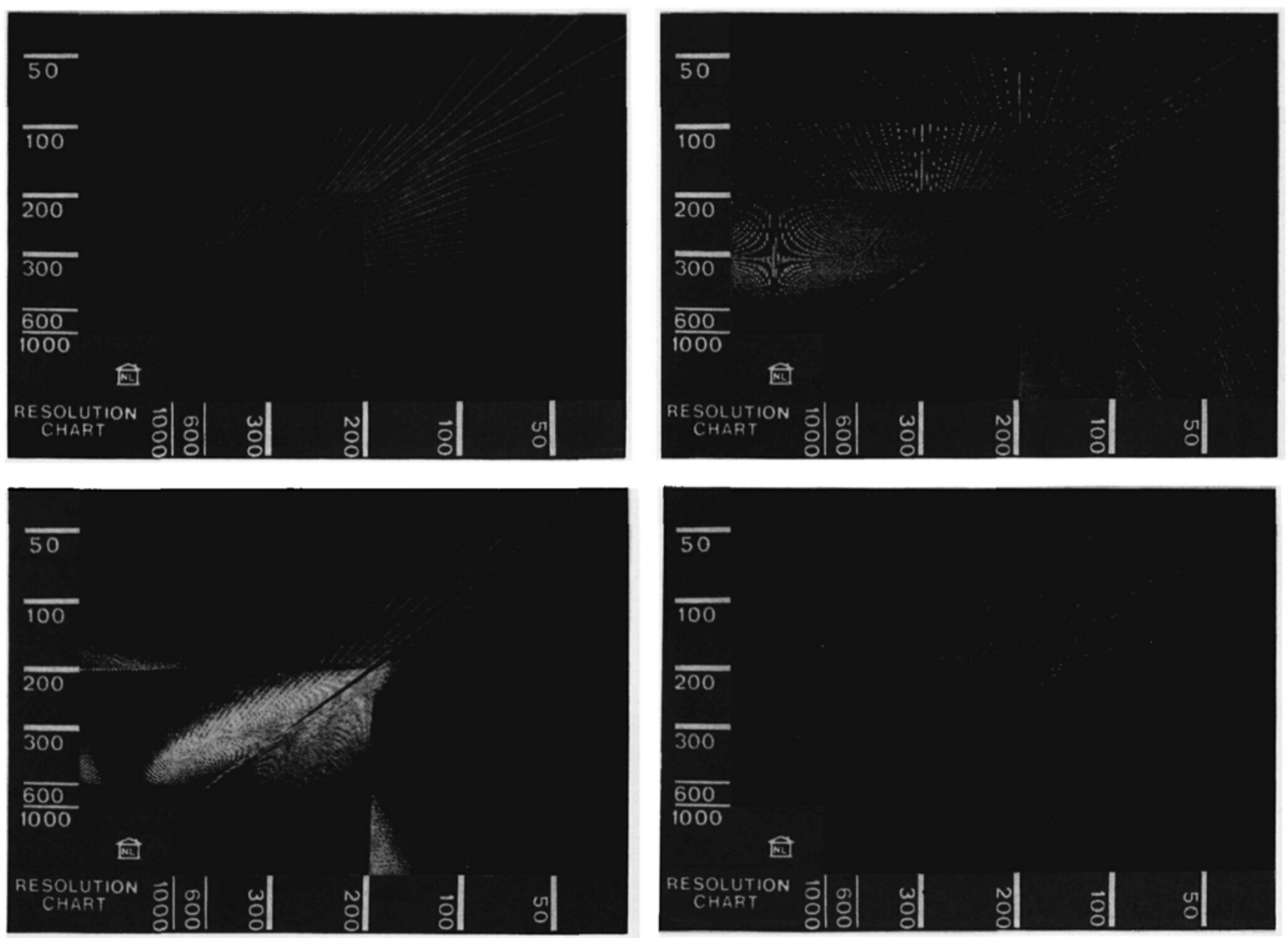

Bild 4: Betrag des Quantisierungsfehlers (multipliziert mit 8) in Bild 3C bei einer Codierung mit 4 bit/Abtastwert für die Prädiktoren 1 bis 4

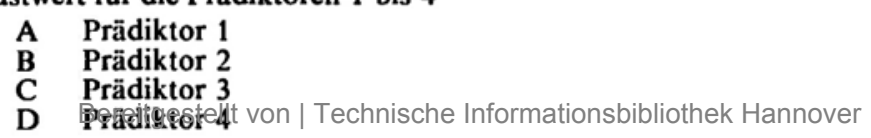


ser Spitzenwert bedeutend geringer. Dies gilt besonders bei konturreichen Bildern. Bei Prädiktor 4 ist für das Auflösungstestbild dieser Spitzenwert um etwa 20 kleiner als bei den anderen Prädiktoren. Diese Verkleinerung hat große Bedeutung für die Verhinderung des slope overload und edge busyness, und demgemäß auch für die Reduzierung der erforderlichen Anzahl von Quantisierungsstufen. Tabelle 6 zeigt ferner die Standardabweichung $\sigma_{\mathrm{f}}$ der Prädiktionsfehler und Tabelle 7 die Standardabweichung $\sigma_{\mathrm{q}}$ der Quantisierungsfehler bei der 4-bit-Quantisierung. Bezüglich der Standardabweichungen ist Prädiktor 4 am günstigsten. Prädiktor 3 ist nicht so günstig, da er außer kleinen Prädiktionsfehlern auch viele große Fehler verursacht. Die Bildabhängigkeit der nichtadaptiven Prädiktoren ist eindeutig erkennbar (z. B. Prädiktor 1 und 2 bei Bild 3B und Bild 3C).

Wie die Tabellen zeigen, hat die Quantisierung keinen bedeutenden Einfluß auf die Vergleichsergebnisse. Da die Unterschiede zwischen Originalen und rekonstruierten Bildern auf einer Fotografie schwer zu erkennen sind, stellen wir in Bild 4 nur dic Quantisierungsfehler dar. Weil der Monitor nur positive Signalwerte anzeigen kann, zeigt Bild 4 den achtfachen Betrag des Quantisierungsfehlers $q$. Die Fotographien zeigen eindeutig den Vorteil des neuen adaptiven Prädiktors.

\subsection{Subjektive Vergleiche}

Die Bildqualität wurde auch durch subjektive Tests verglichen, wie sie von Pirsch [8] vorgeschlagen werden. Nach subjektiven Vergleichen ergibt sich folgende Rangfolge für die vier Prädiktoren beim Auflösungstestbild:

1. Prädiktor 4,

2. Prädiktor 1,

3. Prädiktor 3,

4. Prädiktor 2.

Der Betrag des höchsten benötigten Quantisierungsrepräsentativwertes für das Auflösungstestbild, welcher durch Überprüfungen sowohl von Bildschärfe als auch von Moiré-Störungen festgestellt wird, kann von 104 bei Prädiktor 2 bzw. 72 bei Prädiktor 1 auf 62 bei Prädiktor 4 gesenkt werden. Bei gleicher Bildqualität kann daher mit Prädiktor 4 die Anzahl der Quantisierungsstufen verringert werden. Wie bereits berichtet [4], erreicht Prädiktor 2 bei 29-Stufen-Quantisierung bzw. Prädiktor 1 bei 21-Stufen-Quantisierung eine gute Bildqualität für Auflösungstestbilder. Hierzu spart der neue adaptive Prädiktor 11 bzw. 3 Quantisierungsstufen. Bei einer 16-Stufen-Quantisierung nach Tabelle 1 sind die von slope overload verursachten Moiré-Störungen bei Prädiktor 2 und 3 deutlich sichtbar und bei Prädiktor 1 gerade wahrnehmbar, bei Prädiktor 4 nicht sichtbar. Für natürliche Bilder erhält man mit 4 bit je Abtastwert (16-Stufen-Quantisierung) bei Prädiktor 1, 2 und 4 eine in etwa gleich gute Bildqualität; das Quantisierungsrauschen bei Prädiktor 3 ist größer und gut sichtbar. Mit Prädiktor 4 kann man also bei einer 16-Stufen-Quantisierung eine höhere Bildqualität sowohl für natürliche Bilder als auch für konturreiche Auflösungstestbilder erreichen.

Die mit Prädiktor 3 erreichbare Bildqualität bei 4 bit je Abtastwert ist nicht zufriedenstellend. Hierfür wurden zwei Ursachen gefunden: Erstens ist die Entscheidungsbedingung für horizontale Konturen zu einfach, und damit wird $s_{1}$ zu häufig als Prädiktionswert benutzt [9]; zweitens sind die Umschaltungen verschiedener Prädiktoren zu „hart".

\section{Zusammenfassung}

Ein neuer adaptiver Prädiktor für die DPCM-Intraframe-Codierung von Fernsehsignalen wurde vorgeschlagen und mit bekannten Prädiktoren verglichen. Es wurde gezeigt, daß der neue Prädiktor bessere Prädiktionsfähigkeit als die bekannten Prädiktoren hat. Dies wurde sowohl durch objektive quantitative Vergleiche als auch durch subjektive Tests bestätigt. Der vorgeschlagene Prädiktor bringt vor allem eine Verbesserung für konturreiche Auflösungstestbilder. Damit kann eine höhere Bildqualität gleichzeitig für verschiedene Arten von Bildern bei einem festen Quantisierer mit 4 bit je Abtastwert erreicht werden. Der neue Prädiktor führt auf vergleichsweise wesentlich weniger große Prädiktionsfehler und vermeidet deshalb slope overload und edge busyness bei einer niedrigen Übertragungsbitrate. Die subjektiven Tests wurden mit einer Bildsequenz von 16 aufeinanderfolgenden Teilbildern durchgeführt. Eine durch Quantisierungsfehler oder Signalquellenrauschen verursachte falsche Prädiktorumschaltung wird beim neuen Prädiktor durch spezielle Schaltkriterien reduziert. Die durch falsche Prädiktorumschaltung verursachten Quantisierungseffekte werden dadurch verringert. Zukünftige Untersuchungen sollen auf adaptive Intra-Interframe Codierungen gerichtet sein, da dies eine weitere Reduktion der Übertragungsbitrate bei hoher Bildqualität verspricht.

Die corliegende Arbeit entstand am Institut für Theoretische Nachrichtentechnik und Informationsterarbeitung der Universität Hannover. Herrn Prof. Dr.-Ing. Musmann gilt mein besonderer Dank für die wertvollen Anregungen, ebenfalls Herrn Dr.-Ing. P. Pirsch für hilfreiche Diskussionen. Herrn D. Westerkamp und Herrn W. Geuen danke ich für die freundliche Hilfe bei den Computersimulationen.

\section{Literatur}

II] ONeal, J. B.: Predictive Quantizing Systems. Bell Syst. Techn. J. 45 (1965) S. $689-721$.

12] Habibi, A.: Comparison of nth-Order DPCM Encoder with Linear Transformations and Btock Qumatization Techniques. IEEE Trans. on Comm. COM-19 (1971) 12, 948-956.

[3] Pirsch. P.: Stenger, L.: Statistical Analysis and Coding of Color Video Signals. Acta Electronica 19 (1976) 4, S. $277-287$.

4] Pirsch, P.: A New Predictor Design for DPCMI Coding of TV Signals. ICC (1980). | Zschunke, W.: DPCM Picture Coding with Adaptive Prediction. IEEE Trans. on Comm. CON-25 (1977) 11, S. $1295-1302$.

16] Zschunke, W.: System zu digitalen C'bertragung von Bildsignalen mittels DifferenzPulscodemodulation (DPCM). Bundesrepubilik Deutschland Patent 2432399.

17) Musmann, H. G.: Predictive Image Coding in: Image Transmission Techniques. (ed. by Pratt, W. K.) Academic Press, New York (1979).

[8] Pirsch, P.: Optimierung von Farbfernseb-DPCM-Systemen unter Bericksichtigun der Wahrnehmbarkeit von Quantisierungsfehlern. Dissertation, Universität Hannover (1979).

9] Venkat Deiarajan; Rao, K. R.: DPCM Coders with Adaptive Prediction for NTSC Composite TV Signals. IEEE Trans. on Comm. COM-28 (1980) 7, S. 1079-1084.

Chun-tian Zhang, Dozent an der Universität Tianjin, VR China, zur Zeit als Gastwissenschaftler an der Universität Hannorer, Institut firi Theoretische Nachrichtentechnik und Informationsserarbeitung, Callinstr. 32, 3000 Hannover 1.

(Kingegangen am 18. 12. 1981)

\section{Berichtigung}

In dem Beitrag von U. Barabas „Optische Breitbandübertragung mit Hilfe pulsphasenmodulierter Signale“ (Heft 3/82, S. 69) ist der letzte Absatz der Einleitung durch Zeilenvertauschung falsch wiedergegeben worden. Er muß richtig lauten:

Im folgenden sind drei auf Fernsehsignale angewandte Übertragungsverfahren beschrieben. Anschließend werden mögliche Anwendungsbereiche diskutiert und aus realisierten Sysiemen Mieỉergebnisse angegeber. 